\title{
A Review on Antioxidant, Anti-Inflammatory and Gastroprotective Abilities of Man- go (Magnifera indica) Leaf Extract and Mangiferin
}

\author{
Stohs $\mathrm{SJ}^{*}$, Swaroop $\mathrm{A}^{2}$, Moriyama $\mathrm{H}^{3}$, Bagchi $\mathrm{M}^{2}$, Ahmad $\mathrm{T}^{2}$, and Bagchi $\mathrm{D}^{2,4}$ \\ ${ }^{1}$ Creighton University Medical Center, Omaha, Nebraska, USA \\ ${ }^{2}$ Cepham Research Center, Somerset, New Jersey, USA \\ ${ }^{3}$ The Japanese Institute for Health Food Standards, Bunkyo-ku, Tokyo, Japan \\ ${ }^{4}$ Departments of Pharmacological and Pharmaceutical Sciences, University of Houston College of \\ Pharmacy, Houston, Texas, USA
}

${ }^{*}$ Corresponding author: Stohs SJ, Emeritus Dean, Creighton University Medical Center 2500 California Plaza, Omaha, NE 68178, Nebraska, USA, E-mail: sid.stohs9@gmail.com

Citation: Stohs SJ, Swaroop A, Moriyama H, Bagchi M, Ahmad T, et al. (2018) A Review on Antioxidant, AntiInflammatory and Gastroprotective Abilities of Mango (Magnifera indica) Leaf Extract and Mangiferin. J Nutr Health Sci 5(3): 303. doi: 10.15744/2393-9060.5.303

Received Date: May 18, 2018 Accepted Date: September 11, 2018 Published Date: September 13, 2018

\begin{abstract}
Mango (Mangifera indica Linn.) trees have been cultivated and grown in Southern and Eastern India for thousands of years and have slowly spread all over the world. For over 4,000 years various parts of mango trees including leaf and fruit pulp have been extensively used in Ayurvedic and Unani medicines. Mango pulp and leaves contain diverse health promoting chemical constituents including mangiferin, a natural polyphenol of C-glycosylxanthone structure, with diverse pharmacological/physiological activities including the promotion of gut health and regularity, as well as antioxidant and anti-inflammatory benefits. This review provides a detailed discussion of the nutritional benefits of mango leaf extract and mangiferin in promoting anti-inflammatory, antioxidant, gastrointestinal and gut health benefits. In addition, information regarding the tissue protective, immunomodulatory, antidiabetic, anti-hyperlipidemic and anti-cancer properties, as well as for disease management and health benefits of mango leaf extracts and mangiferin will be reviewed. Furthermore, mechanistic studies involving the free radical scavenging ability of mangiferin as well as its ability to exert antiinflammatory activity by inhibiting the expression of the TNF- $\alpha$ and related signaling mechanisms and inducible nitric oxide synthase will be considered.

Keywords: Mango Fruit and Leaves; Mangiferin; Anti-inflammatory; Antioxidant; Gastro-Intestinal and Gut Health; Regularity

List of abbreviations: ALT: Alanine Aminotransferase; AMP: Adenosine Monophosphate; AMPK: AMP Activated Protein Kinase; ASC: Apoptosis-associated Speck-Like Protein Containing a Caspase-Recruitment Domain; AST: Aspartate Aminotransferase; ATP: Adenosine Triphosphate; Bcl-2: B-cell Lymphoma 2; BUN: Blood Urea Nitrogen; CD73: Cluster of Differentiation 73; DOX: Doxorubicin; DPPH: 1,1-diphenyl-2-picrylhydrazyl; ERK5: Extracellular-signal-regulated kinase 5; GTP: Guanosine-5'-triphosphate; HO-1: Hemeoxygenase-1; IBD: Inflammatory Bowel Disease; ICAM-1: Intercellular Cell Adhesion Molecule-1; IKBa: Nuclear Factor of Kappa Light Polypeptide Gene Enhancer in B-cells Inhibitor, Alpha; IL-1 $\beta$ : Interleukin-1beta; IL-6: Interleukin 6; LPS: Lipopolysaccharide; MAPK: Mitogen-activated Protein Kinase; MCP-1: Monocyte Chemoattractant Protein-1; MDA: Malondialdehyde; MMP-9: Matrix Metallopeptidase 9; MPO: Myeloperoxidase; NF-K $\beta$ : Nuclear Factor Kappa-light-chain-enhancer of Activated B Cells; NLRP3: Nucleotidebinding domain, Leucine-rich-containing Family, Pyrin Domain-containing-3; NMR: Nuclear Magnetic Resonance; NO: Nitric Oxide; RANTES: Regulated on Activation, Normal T Cell Expressed and Secreted; TBARS: Thiobarbituric Acid Reactive Substances; TNF- $\alpha$ : Tumor Necrosis Factor Alpha; TNBS: 2,4,6-Trinitrobenzensulfonic Acid; TPGS: D- $\alpha$-Tocopherol Polyethylene Glycol 1000 Succinate
\end{abstract}

\section{Introduction}

According to Ayurveda and indigenous medical systems, mango [Mangifera indica Linn. family Anacardiaceae] is a tropical fruit with a distinctive nutritional and phytochemical composition [1,2]. Earlier literature recommends mango leaves, bark, seeds, flowers, and raw and ripe fruits for a myriad of medicinal, pharmacological and health benefits [1-5]. In addition, mango leaf is considered holy and sacred, and used as an integral part of all Hindu rituals from the Vedic ages. Research studies indicate that mango leaves and fruits exhibit antioxidant, anti-inflammatory, anti-diabetic, cardioprotective, anti-tumor, wound healing, anti-pyretic, anti-bacterial, anti-spasmodic, anti-carcinogenic, anti-viral, anti-microbial and anti-dysentry benefits, as well as 
hepatoprotective, gastroprotective, immunomodulatory and hypolipidemic effects [1-5]. The juice of ripe mango is purported to be beneficial against heat stroke, a fatal life-threatening inflammatory response $[1,2,4,5]$.

Mango trees have been cultivated in the Indian sub-continent for thousands of years. They have been grown in Southeast Asia from the fifth and fourth centuries BC, and have been cultivated in East and West Africa since around the 10th century AD. Subsequently, cultivation of mangoes has occurred in Brazil, Mexico, West Indies and Caribbean Islands [6,7]. Mangos are also grown in Southern Europe, especially in the coastal subtropical environment of Spain, Canary Islands and Portugal. Alphonso mango is the most popular mango species grown in India, which is now exported worldwide as a fruit or juice. In 2012, the United Nations statistics indicated that India is the largest producer of mango (16.2 million tons), while China and Thailand produced approximately 4.4 and 3.0 million tons, respectively $[1,6,7]$.

Mangoes are also used for various culinary purposes. Raw mangoes are sour and used in pickles and salad, while ripe mangoes are generally sweet and used in yogurt, juices, smoothies, pies, and milk shakes $[1,2,8]$. The purpose of this review is to provide an assessment primarily of the gastrointestinal effects of mango leaf extracts and mangiferin, the primary active constituent thereof, as well as their mechanisms of action.

\section{Mango and Mango Leaf Constituents ${ }^{1}$}

Mango leaves has been demonstrated to contain mangiferin (Figure 1), selected anthocyanidins including delphinidin, peonidin and cyanidin, leucoanthocyanins, catechin and gallic tannins, the mangiferin content of mango leaves ranges from about 2-15\% depending on the variety and geographic source, while extracts of mango leaves contain up to approximately $60 \%$ mangiferin. Mango leaf oil is rich in sesquiterpenes and also contains mangiferin, $\delta$-3-carene, $\alpha$-gurjunene, $\beta$-selinene and $\beta$-caryophyllene. A phytochemical analysis reported that mango leaves contain alkaloids $(0.84 \pm 0.11 \mathrm{mg} / 100 \mathrm{~g})$, phenols $(0.09 \pm 0.20 \mathrm{mg} / 100 \mathrm{~g})$, flavonoids $(11.24 \pm 0.10 \mathrm{mg} / 100 \mathrm{~g})$, saponins $(3.22 \pm 0.10 \mathrm{mg} / 100 \mathrm{~g})$ and tannins $(0.45 \pm 0.10 \mathrm{mg} / 100 \mathrm{~g})[9,10]$. Thus, mango leaves contain high amounts of polyphenolic antioxidants including xanthonoids, mangiferin and gallic acid $[9,10]$.

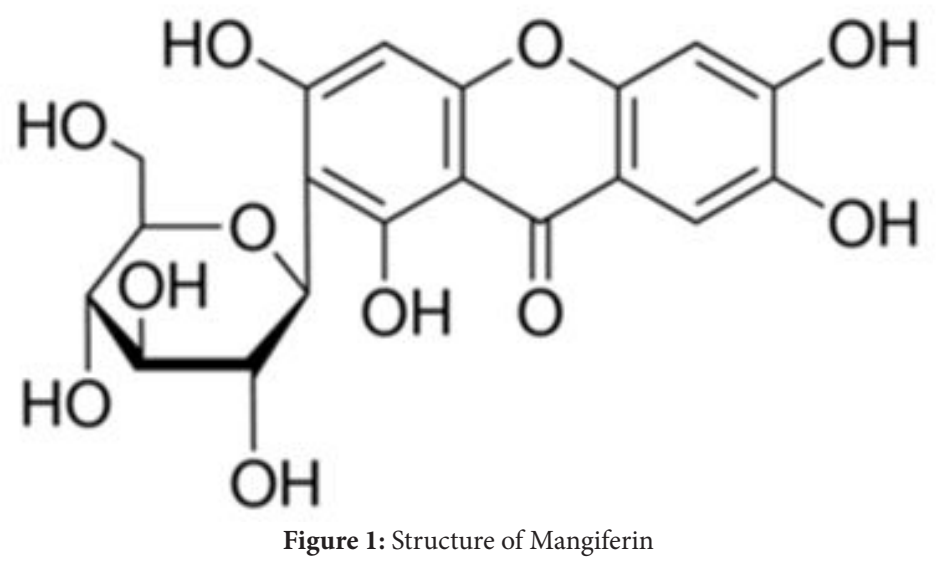

Yusukol et al. developed an enzyme-linked immunosorbent assay [ELISA] for mangiferin and examined the mangiferin content of the mature leaves of 50 mango cultivars. The mangiferin content ranged from 1.94 to $13.79 \mathrm{mg} / \mathrm{g}$ (\%) [11]. An Indian Thawai cultivar exhibited the highest content while a Namdokmai cultivar from Thailand contained $12.41 \mathrm{mg} / \mathrm{g}$. Sharma et al. assessed the mangiferin content of aqueous extracts of 30 varieties of mango from west India using HPLC and UV detection [12]. The average mangiferin content from these varieties was $17.5 \mathrm{mg} / \mathrm{g}$ with the highest content from a Ladvo variety being $47.02 \mathrm{mg} / \mathrm{g}$.

Mango leaves have also been shown to contain calcium $(3.82 \pm 0.10 \mathrm{mg} / 100 \mathrm{~g})$, magnesium $(0.91 \pm 0.20 \mathrm{mg} / 100 \mathrm{~g})$, potassium $(0.83 \pm 0.10 \mathrm{mg} / 100 \mathrm{~g})$, sodium $(0.38 \pm 0.11 \mathrm{mg} / 100 \mathrm{~g})$, zinc $(7.88 \pm 0.10 \mathrm{mg} / 100 \mathrm{~g})$, cadmium $(1.50 \pm 0.20 \mathrm{mg} / 100 \mathrm{~g})$, copper $(8.68 \pm 0.10 \mathrm{mg} / 100 \mathrm{~g})$ and phosphorus $(0.78 \pm 0.10 \mathrm{mg} / 100 \mathrm{~g})$ [1]. Furthermore, mango leaves are a good source of ascorbic acid $(29.92 \pm 0.11 \mathrm{mg} / 100 \mathrm{~g})$, riboflavin $(0.09 \pm 0.10 \mathrm{mg} / 100 \mathrm{~g})$, niacin $(0.75 \pm 0.20 \mathrm{mg} / 100 \mathrm{~g})$ and thiamine $(0.45 \pm 0.11 \mathrm{mg} / 100 \mathrm{~g})[9]$.

\section{Chemistry and Structure of Mangiferin}

A growing body of evidence suggests the significant potential of mangiferin (2C- $\beta$-D-glucopyranosyl-1,3,6,7-tetrahydroxyxanthone; molecular formula $\mathrm{C}_{19} \mathrm{H}_{18} \mathrm{O}_{11}$; CAS 4773-96-0; molecular weight 422.34), a xanthone-C-glycoside present in diverse plant species, for versatile anti-inflammatory responses, potent antioxidant, radioprotective, immunomodulatory and anti-cancer properties, as well as for disease management and health benefits [8-21]. The antioxidant efficacy of mangiferin is stronger than cimmamon [1,13-16]. Furthermore, mangiferin exerts a pro-hypoglycemic activity by modulating glucose metabolism, ameliorating insulin resistance, lowering cholesterol synthesis, and inhibiting the expression of the TNF- $\alpha$ and inducible nitric oxide synthase [13-21].

Chemically, mangiferin is composed of a glucose unit $\mathrm{C}_{1 \rightarrow 2}$ chemically bonded to a 1,3,6,7-tetrahydroxyxanthone aglycone, and lately it has been recognized as a potential pharmacophore. It is a light yellow color crystalline powder, slightly soluble in ethyl 
alcohol, sparingly soluble in methyl alcohol and water, and insoluble in diethyl ether, acetone and hexane $[1,13,14]$. It has also been reported that the solubility of mangiferin decreases with increasing temperature in the order ethanol $>$ methanol $>$ water $>$ diethyl ether $>$ acetone $>n$-hexane $[1,10]$. Somewhat in contrast to these observations, the ability of high pressure techniques to extract mangiferin lead to the following results [22]. Extraction of leaves with supercritical $\mathrm{CO}_{2}$ results in mangiferin extraction of about $1 \mathrm{mg} / \mathrm{g}$, while adding ethanol to supercritical $\mathrm{CO}_{2}$ increases the yield to an average of $6.5 \mathrm{mg} / \mathrm{g}$. However, when using a pressurized water extraction at $100{ }^{\circ} \mathrm{C}$ for 3 hours, the average yield was approximately $29 \mathrm{mg} / \mathrm{g}$, providing an economically feasible system for extraction of mangiferin on an industrial scale.

\section{Safety and Toxicity Studies of Mango Leaf Extract}

A 14 day acute oral toxicity study of an aqueous ethanol extract of dried mango leaves standardized to $62 \%$ mangiferin was conducted in 40 male and female ICR mice (body weight 18-21 grams) [23]. No signs of toxicity or toxic manifestations were observed at maximum oral dose of $18.4 \mathrm{~g}$ mango leaf extract $/ \mathrm{kg}$ bodyweight $(11.4 \mathrm{~g}$ mangiferin $/ \mathrm{kg})$ twice a day with a $4 \mathrm{~h}$ interval between dosing. The animals were observed for 14 days for toxic reactions and mortality. At the end of the 14 days, all animals were healthy with no abnormal responses or lesions [23].

Another study demonstrated no signs of toxicity at a single oral dose of 5,000 mg of a methanol mango leaf extract $/ \mathrm{kg}$ body weight, which demonstrates its broad spectrum of safety [24]. The extract contained approximately $4.25 \%$ mangiferin. The animals were observed for 14 days and no signs of gross toxicities including toxic manifestations or mortality were observed. Animals were also executed for gross anatomy check and macroscopic evaluation and no obvious organ changes were observed [24].

In a sub-chronic 90-day toxicity study, forty-eight male and female Sprague-Dawley rats (body weight 117-160 g) were orally administered either $0,100,300$ or $900 \mathrm{mg}$ of an aqueous ethanol mango leaf extract/kg body weight daily, which was equivalent to 17.2, 51.7 and 155.2 times the clinical daily dose $(5.8 \mathrm{mg} / \mathrm{kg})$, respectively, over a period of 90 consecutive days [23]. The extract contained $62 \%$ mangiferin. No significant abnormalities were observed in body weight and feed intake. All animals were normal at all doses with respect to coagulation functions, gross anatomy, hematology, biochemical criteria, and relative organ weights. Small but higher triglyceride and cholesterol levels were observed at the high dose $(900 \mathrm{mg} / \mathrm{kg})$ in male rats, and a lower potassium level was detected in female rats in the high dose group. Overall, no significant adverse events were observed [23].

Although no direct mutagenicity studies have been reported on mango leaf extract, based on its huge consumption by animals and humans around the world for several centuries, it can be considered non-mutagenic. A detailed investigation by Gold-Smith demonstrated the novel anti-cancer properties of mangiferin, which further supports its non-mutagenic potential [14].

\section{Antioxidant Benefits of Mangiferin}

Various research studies have demonstrated that mango leaf extract and mangiferin exhibit potent antioxidant and free radical scavenging activities, and the antioxidant potential is remarkably amplified in pro-inflammatory and inflammatory conditions, including infection and diabetic states [25-37]. The four hydroxyl groups in mangiferin demonstrate remarkable antioxidant properties by scavenging the noxious oxygen free radicals. As a potent iron chelator, it prevents the production of noxious hydroxyl radicals in Fenton-type reactions, as well as protects against UV (ultraviolet) radiation [18]. Using the DPPH assay, Dar et al. demonstrated the potent antioxidant activity of mangiferin $\left(\mathrm{EC}_{50} 5.8 \pm 0.96 \mu \mathrm{g} / \mathrm{ml}\right.$ ) which is comparable to rutin, a potent antioxidant used for medical purposes [27]. It lowers hydrogen peroxide-induced lipid peroxidation in human peripheral blood lymphocytes in a dose-dependent manner. It prevents the depletion of GTP and total nucleotides as well as ATP damage and restores energy charge potential in $\mathrm{H}_{2} \mathrm{O}_{2}$-treated erythrocytes [25-29]. It also reduces diabetes-induced lipid peroxidation (TBARS) to a healthy level, as well as exhibiting antidiabetic activity [13,30].

Pardo-Andreu et al. showed that mangiferin works by a unique mechanism which is quite different from the classical hydroxyl radical scavengers, retaining iron in its original ferric form and by complexing $\mathrm{Fe}^{3+}$ or stimulating $\mathrm{Fe}^{2+}$ autoxidation [28-31]. However, Pokorski et al. in a hypoxic ventilator response model in rats indicated that the antioxidant properties superceded the iron chelating properties in this system [14]. Overall, the antioxidant potential of mangiferin counterbalances the iron-induced oxygen free radical formation, $\mathrm{Fe}^{2+}$ accumulation and iron-induced toxicity [28-31].

Various studies have affirmed the hepatoprotective effects of mango leaf extract and mangiferin. Karuppanan et al. demonstrated the hepatoprotective and antioxidant effects of mango leaf extracts against mercury chloride-induced liver toxicity in mice [32]. Extracts were shown to increase hepatic catalase, glutathione S-transferase and glutathione peroxidase levels. A methanolic extract was more effective than an ethanolic extract. Mangiferin has been shown to be a potent hepatoprotective against carbon tetrachloride-induced liver injury, which further supports its free radical scavenging activities [27]. Mangiferin protects against ethanol-induced hepatic damage [33]. Mangiferin also exhibited anti-apoptotic activity and anti-oxidant cytoprotection against glutaminergic excitotoxicity leading to neuronal death [34]. Arsenic-induced oxidative stress in the hepatic tissues and liver dysfunction was also dramatically protected by mangiferin in another study [32].

Xia et al. investigated the protective ability of mangiferin in a molecular model of $\mathrm{H}_{2} \mathrm{O}_{2}$-induced oxidative injury in osteoblast-like cultured MC3T3-E1 cells [35]. Mangiferin significantly reduced $\mathrm{H}_{2} \mathrm{O}_{2}$-induced apoptosis and oxidative stress in the MC3T3-E1 
cells. The study demonstrated that mangiferin can protect these cultured MC3T3-E1 cells against oxidative injury by modulation of ERK5/Nrf2 signaling. These results suggest that mangiferin may be a potential agent in the intervention and prevention of osteoporosis [35].

\section{Anti-Inflammatory Benefits of Mangiferin}

A number of research studies have demonstrated that mangiferin exerts anti-inflammatory potential in various models. Pan et al. investigated the molecular mechanism of mangiferin against lipopolysaccharide (LPS) and D-galactosamine-induced acute liver injury and inflammation [36]. Mangiferin inhibited the levels of serum ALT, AST, IL-1 $\beta$, TNF- $\alpha$, MCP-1, and RANTES (regulated on activation, normal $\mathrm{T}$ cell expressed and secreted), as well as hepatic lipid peroxidation and oxidative stress. Mangiferin also inhibited IL-1 $\beta$ and TNF- $\alpha$ production in LPS-stimulated primary hepatocytes. Mangiferin up-regulated the expression of Nrf2 and HO-1 in a dose-dependent manner. Furthermore, mangiferin inhibited LPS/d-GalN-induced hepatic NLRP3, ASC, caspase-1, IL- $1 \beta$ and TNF- $\alpha$ expression. Thus, mangiferin protected against the liver injury by activating the Nrf2 pathway and regulating NLRP3 inflammasome activation [36].

Pardo-Andreu et al. have shown that mangiferin dose-dependently inhibited the inflammatory cytokines, TNF $\alpha, \mathrm{NO}$ and NF-K $\beta$ in both in vitro and in vivo models, affirming the anti-inflammatory potential of mangiferin [28]. Saha et al. postulated that the $\mathrm{C}$-glucosyl linkage and hydroxyl groups in mangiferin contribute significantly to its free radical-scavenging activity. Furthermore, it modulates various transcription factors including NF- $\mathrm{kB}$ and Nrf-2, and expression of multiple pro-inflammatory signaling molecules including TNF- $\alpha$ and COX-2, which contribute to its anti-inflammatory benefits [17,37].

Agustini et al. demonstrated the protective abilities of mangiferin against doxorubicin (DOX)-induced cardiotoxicity and inflammatory responses in rats [38]. Mangiferin exhibited protection against DOX-induced cardiac inflammation and apoptosis via down-regulation of pro-apoptotic and pro-inflammatory gene expressions, upregulation of SERCA2a gene expression, and normalization of cytosolic calcium levels [38].

Wang et al. showed that mangiferin protected against renal ischemia-reperfusion injury by inhibiting inflammation, oxidative stress and inducing adenosine production in a mice model [39]. Mangiferin improved kidney function, increased CD73 expression in kidneys suffering from ischemia-reperfusion injury, enhanced adenosine production, and inhibited pro-inflammatory responses and tubular apoptosis [39].

Gong et al. demonstrated that mangiferin can attenuate cecal ligation and puncture-induced mortality and acute lung injury (ALI), as indicated by reduced systemic and pulmonary inflammatory responses as exhibited by reduced systemic and pulmonary inflammatory responses [40]. Mangiferin inhibited sepsis-activated mitogen-activated protein kinases (MAPK) and NF-K $\beta$ signaling, demonstrating the inhibition of pro-inflammatory mediators. Furthermore, mangiferin dose-dependently up-regulated HO-1 in the pulmonary tissues of septic mice. It was further concluded that mangiferin attenuated sepsis by up-regulation of HO1, which protects against sepsis-induced acute lung injury by modulating inflammatory responses [40].

Szandruk et al. assessed the protective ability of mangiferin in colon tissues against 2,4,6-trinitrobenzensulfonic acid (TNBS)induced colitis in rodents [41]. Mangiferin reduced macroscopic and microscopic damage, as well as reduced MDA levels in the colon at 30 and $100 \mathrm{mg} / \mathrm{kg}$ body weight doses. Mangiferin $(100 \mathrm{mg} / \mathrm{kg}$ body weight) also reduced TNF- $\alpha$ and IL-17 levels, and SOD activity in the colon tissues. The researchers concluded that mangiferin significantly protects against TNBS-induced inflammatory manifestations and colitis in rats by potentiating anti-inflammatory and antioxidant activities [41].

\section{Gastrointestinal Transit and Gut Health}

Human bacterial enzymes have been shown to metabolize mangiferin into phenolic acid metabolites in the colon, where these metabolites are capable of exhibiting biochemical and pharmacological effects [42]. Huang et al. demonstrated by using mass spectrometry and NMR that mangiferin can be metabolized in vitro by human intestinal bacteria into its corresponding aglycone 1,3,6,7-tetrahydroxyxanthene [42]. Bock and Ternes administered $74 \mathrm{mg}$ mangiferin/kg body weight to pigs/day over a period of 11 days and feces were collected on day 11 and analyzed [43]. The researchers demonstrated that phenolic acids excreted in the feces included 3,4-dihydroxybenzoic acid, 3,4-dihydroxy-phenylacetic acid, 2,4,6-trihydroxybenzoic acid, and 3,4,5-trihydroxybenzoic acid following metabolism by intestinal microflora of animals treated with mangiferin.

In another study, Sanugul et al. identified the anaerobic bacteroides MANG which is involved in mangiferin metabolism to its corresponding aglycone through cleavage of its C-glucosyl bond [44]. MANG cleaved C-glucosyl in a dose- and timedependent manner only when cultivated in the presence of mangiferin. However, mangiferin did not affect bacterial alpha- and beta-glucosidase activities under any conditions. The researchers pointed out that the $\mathrm{C}$-glucosyl-cleaving enzyme substantially differs from known glucosidases that cleave O-glucosides. Furthermore, the researchers claimed that this is the first description of a specific intestinal bacterium that is involved in the metabolism of mangiferin and which produces a novel and inducible C-glucosyl-cleaving enzyme [44].

Helicobacter pylori-induced gastrointestinal injury including gastric cancer is a challenging problem and no appropriate 
therapeutic intervention has been developed. Zhang and Yue demonstrated that mangiferin has the ability to eradicate $\mathrm{H}$. pylori and concurrently inhibit multiple inflammatory markers including NF-K $\beta$ subunit p65, IL-1 $\beta$, IL- 8 and TNF- $\alpha$. In addition, the protein expression of inflammatory enzymes as COX-2 and iNOS were remarkably down-regulated in cultured human gastric carcinoma AGS cells following incubation with mangiferin [45]. The researchers concluded that mangiferin significantly downregulated the adhesion and invasion process as well as deactivated NF-p65, thereby blocking the inflammatory response and thus reducing the incidence of gastric carcinoma [45]. Interestingly, in several other studies, mangiferin has demonstrated inhibitory activity against Helicobacter pylori-induced inflammatory responses in the gastrointestinal tissues and gastrointestinal toxicity $[42-44,46]$.

Morais et al. investigated the dose-dependent beneficial effects of mangiferin on gastrointestinal transit in normal and constipated mice and the underlying mechanism [47]. Overnight starved Swiss mice were orally administered 3, 10, 40 or $100 \mathrm{mg}$ mangiferin/ $\mathrm{kg}$ body weight or the drug tegaserod ( $1 \mathrm{mg} / \mathrm{kg}$ body weight, intraperitoneally) $30 \mathrm{~min}$ before a charcoal meal to assess the effect of mangiferin on normal transit. In the second set of experiments, the effect of mangiferin ( $30 \mathrm{mg} / \mathrm{kg}$ body weight) on delayed gastrointestinal transit induced by selected agonist(s) or antagonist(s) were assessed [47]. In the third set of experiments, mice were orally administered 3, 10, 40 or $100 \mathrm{mg}$ mangiferin/kg body and 6 hour fecal pellet output was assessed. In addition, the ratio of wet to dry weight was calculated and used as a marker of fecal water content. The researchers concluded that mangiferin accelerated gastrointestinal transit in both normal and constipated mice and enhanced fecal output, but not water content in the fecal mass, while in the tegaserod group the fecal pellets had higher water content. The authors indicated that the increase in water content is indicative of augmented secretary activity. Overall, mangiferin was shown to have a safe prokinetic profile, since no diarrhea inducing effect was observed. The researchers concluded that mangiferin may serve as a functional agent to enhance regularity and ameliorate gastrointestinal disturbances [47]. Incidentally, an aqueous decoction of mango leaves enriched in polyphenols including mangiferin demonstrated potent antiulcerogenic potential [26].

Carvalho et al. investigated the gastroprotective efficacy of mangiferin against ethanol and indomethacin-induced gastric mucosal injury in mice. Mean gastric lesion areas and ulcer scores were determined [48]. Mangiferin significantly protected against ethanol-induced depletion of gastric mucosal non-protein sulfhydryl content in mice. Administration of mangiferin at 3, 10 and $30 \mathrm{mg} / \mathrm{kg}$ in mice decreased ethanol-induced gastrointestinal injury significantly by 30, 35 and 63\%, respectively, and also reduced indomethacin-induced gastric injury [48]. Furthermore, mangiferin prevented gastrointestinal protein depletion in the gastric mucosa of rats.

Kakino et al. demonstrated a laxative effect and mechanism of an ethanolic extract of agarwood (Aquilaria sinensis, Aquilaria crasna) leaves, in which one of the major constituent is mangiferin. The authors demonstrated that these agarwood leaves enriched in mangiferin exerted a laxative effect via acetylcholine receptors in a mouse constipation model [49].

Wang et al. indicated that the therapeutic efficiency of mangiferin is restricted by its low intestinal permeability, and in order to improve the oral absorption of mangiferin, the authors assessed the comparative in vivo efficacy using a number of potential absorption enhancers including D- $\boldsymbol{a}$-tocopherol polyethylene glycol 1000 succinate (TPGS), sodium deoxycholate and Carbopol 974P [50]. Increased oral absorption by the three co-excipients was in the order of Carbopol 974P $>$ sodium deoxycholate $>$ TPGS. The researchers concluded that these compounds are effective in enhancing the absorption of mangiferin in the gastrointestinal tract [50].

Mahmoud-Awny et al. investigated the molecular mechanism of antioxidant gastroprotective ability of mangiferin in a rat model of gastric ulcer. Modulatory effects on the signaling pathway were investigated using ischemia/reperfusion model in animals [51]. Mangiferin produced its gastroprotective benefits partly via inducing the expression of Nrf2, HO- 1 and PPAR- $\gamma$ along with downregulating NF-K $\beta$. The antioxidant benefits were manifested by increasing total antioxidant capacity and reduced glutathione levels, besides normalizing (decreasing) MDA levels. Mangiferin reduced ischemia-reperfusion-induced NO dose dependently, enhanced endothelial nitric oxide synthase and also reduced the inducible isoform of nitric oxide synthase. Mangiferin exerted anti-inflammatory benefits by reducing serum IL-1 $\beta$ and E-selectin levels, effects that were mirrored in the tissue levels of myeloperoxidase, the neutrophil infiltration marker. Furthermore, mangiferin also exerted an anti-apoptotic effect by increasing Bcl-2 levels and reducing caspase-3 in a dose-dependent manner [51].

Another study investigated the gastroprotective benefits of a Mangifera indica leaf decoction in rodents. An aqueous decoction of Mangifera indica extract enriched in mangiferin exhibited significant gastroprotective benefits, including an antiulcerogenic activity [52]. Somani et al. investigated the molecular mechanism of mangiferin in a mouse model of colitis and inflammatory bowel disease (IBD) [53]. A molecular docking model and glide score demonstrated that mangiferin had significant binding potential with TNF- $\alpha$ and MMP-9. The researchers concluded that mangiferin reduced colonic injury in colitis by modulating oxidative and inflammatory parameters partly through attenuating the activity of TNF- $\alpha$ and MMP-9. Therefore, mangiferin may have potential in the management of IBD [53].

Morais et al. investigated the anti-inflammatory and prokinetic actions of mangiferin in a mouse model of post-operative ileus, as well as determined the potential ability of mangiferin to attenuate intestinal inflammation and impaired gastrointestinal transit [54]. These investigators concluded that mangiferin significantly inhibited the myeloperoxidase activity, a biomarker of neutrophil 
infiltration, and nitrate/nitrite levels in the ileum, and decreased the plasma levels of TNF- $\alpha$, IL-1 $\beta$, IL-6, and MCP-1. Thus, mangiferin significantly ameliorated intestinal inflammation and impaired gastrointestinal motility [54].

Dou et al. demonstrated that mangiferin significantly attenuated dextran sulfate sodium (DSS)-induced body weight loss, diarrhea, colon shortening and histological injury, which correlated with the decline in the activity of MPO and the levels of TNF- $\alpha$ in the colon [55]. DSS-induced degradation of I $\mathrm{B} \alpha a$ and the phosphorylation of NF-K $\beta$ p65 as well as the mRNA expression of pro-inflammatory mediator's iNOS, ICAM-1, TNF- $\alpha$, IL-1 $\beta$ and IL- 6 in the colon were also down-regulated by mangiferin. Additionally, the phosphorylation/activation of DSS-induced MAPK proteins was inhibited. The researchers recommended that mangiferin may be utilized in the treatment of human IBD [55].

Herrera-Cazares et al. conducted an interesting investigation to develop a functional confectionery with two prime objectives: (1) retain the integrity of the phenolic antioxidants, and (2) enhance gastrointestinal absorption [56]. A confectionery product was formulated containing $20 \%$ mango bagasse, gelatin and pectin with a mangiferin content of $830.69 \mu \mathrm{g} / \mathrm{g}$, a total polyphenol content $4.14 \mathrm{mg} / \mathrm{g}$, a quercetin content of $244.83 \mu \mathrm{g} / \mathrm{g}$, and a gallic acid content of $285.43 \mu \mathrm{g} / \mathrm{g}$ which was accessible in the intestine after 60 $120 \mathrm{~min}$ of digestion. The results demonstrated that mangiferin exhibited increased bioaccessibility, absorption and antioxidant potential from this confectionery formulation [56].

\section{Conclusions}

A large body of historical Ayurvedic evidence indicates that mango leaf exerts diverse health benefits. A growing body of evidence involving animal, human clinical as well as in vitro studies supports the contention that mango leaf and mango leaf extracts exert diverse health-promoting benefits that may be as effective or superior to other modalities. This review summarizes the diverse health benefits of mango leaf extract enriched in mangiferin in promoting gastrointestinal protection, tissue protection, and glucose as well as lipid regulatory effects. It also eradicates noxious bacteria as Helicobacter pylori, and improves chronic renal and gut health $[42,43]$. Mangiferin exerts its effects by protecting the human body against free radical damage and oxidative stress, hence diminishing membrane damage and inflammation [57]. Additional research and clinical trials are warranted to confirm the diverse health benefits of mangiferin.

\section{Acknowledgements}

The authors thank Ms. Nipa Shah for technical assistance.

\section{References}

1. Shah KA, Patel MB, Patel RJ, Parmar PK (2010) Mangifera Indica (Mango). Pharmacogn Rev 4: 42-8.

2. Burton-Freeman BM, Sandhu AK, Edirisinghe I (2017) Mangos and their bioactive components: adding variety to the fruit plate for health. Food Funct 8: 3010 32.

3. UNCTD (2016) Mango: An Infocomm Commodity Profile UNCTAD Trust Fund on Market Information on Agricultural Commodities. UNCTAD, New York. 4. Imran M, Arshad MS, Butt MS, Kwon JH, Arshad MU (2017) Mangiferin: a natural miracle bioactive compound against lifestyle related disorders. Lipids Health Dis 16: 84 .

5. Gupta N, Jain SK (2014) Storage behavior of mango as affected by post harvest application of plant extracts and storage conditions. J Food Sci Technol 51: 2499507.

6. Glehill D (1972) West African trees, Longman, Hong Kong, China.

7. Oliver-Baver B (1986) Medicinal plants in tropical West Africa. Cambridge University Press, London, UK.

8. Acevedo LM, Raya AI, Martinez-Moreno JM, Aguilera-Tejero E, Rivero JLL (2017) Mangiferin protects against adverse skeletal muscle changes and enhances muscle oxidative capacity in obese rats. PLoS One 12: e0173028.

9. Okwu DE, Ezenagu V (2008) Evaluation of the Phytochemical Composition of Mango (Mangifera Indica Linn) Stem Bark and Leaves. Int J Chem Sci 6: 705-16. 10. Dzamic AM, Marin PD, Gbolade AA, Ristic MS (2010) Chemical Composition of Mangifera indica Essential Oil From Nigeria. J Essen Oil Res 22: 123-5.

11. Yusakul G, Kitirattrakarn W, Tanwanichkul N, Tanaka H, Putalun W (2012) Development and Application of an Enzyme-linked Immunosorbent Assay for Specific Detection of Mangiferin Content in Various Cultivars of Mangifera indica Leaves Using Anti-mangiferin Polyclonal Antibody. J Food Sci 77: 414-9.

12. Sharma BG, Mammen D, Albert S Assessment of Mangiferin in Thirty Varieties of Mangifera Indica L. (Anacardiaceae). Int J Pharmacog 4: 169-73.

13. Pokorski M, Rekawek A, Zasada I, Antosiewicz J, Delgado R (2012) Antioxidation and the hypoxic ventilatory response. Adv Exp Med Biol 758: 373-80.

14. Pokorski M (2013) Mangiferin - A Nutraceutical with Clinical Implications. Vitam Miner 2: e122.

15. Zajac D, Stasinska A, Delgado R, Pokorski M (2013) Mangiferin and its traversal into the brain. Adv Exp Med Biol 756: 105-11.

16. Gold-Smith F, Fernandez A, Bishop K (2016) Mangiferin and Cancer: Mechanisms of Action. Nutrients 8: 396.

17. Saha S, Sadhukhan P, Sil PC (2016) Mangiferin: A xanthonoid with multipotent anti-inflammatory potential. Biofactors 42: 459-74.

18. Benard O, Chi Y (2015) Medicinal properties of mangiferin, structural features, derivative synthesis, pharmacokinetics and biological activities. Mini Rev Med Chem 15: 582-94.

19. Telang M, Dhulap S, Mandhare A, Hirwani R (2013) Therapeutic and cosmetic applications of mangiferin: a patent review. Expert Opin Ther Pat 23: 1561-80. 20. Matkowski A, Kus P, Goralska E, Wozniak D (2013) Mangiferin - a bioactive xanthonoid, not only from mango and not just antioxidant. Mini Rev Med Chem 13: 439-55.

21. Beltran AE, Alvarez Y, Xavier FE, Hernanz R, Rodriguez J, et al. (2004) Eur J Pharmacol 499: 297-305. 
22. Fernandez-Ponce MT, Casas L, Mantell C, Martinez de la Ossa E (2013) Potential Use of Mango Leaves Extracts Obtained by High Pressure Technologies in Cosmetic, Pharmaceutics and Food Industries. J Chem Eng Transact 32: 1147-52.

23. Zhang Y, Li J, Wu Z, Liu E, Shi P, et al. (2014) Acute and Long-Term Toxicity of Mango Leaves Extract in Mice and Rats. Evid Based Complement Altern Med 2014: 691574 .

24. Gururaia GM, Mundkinajeddu D, Kumar AS, Dethe SM, Allan JJ, Agarwal A (2017) Pharmacol Res 9: 21-6.

25. Vyas A, Syeda K, Ahmad A, Padhye S, Sarkar FH (2012) Perspectives on medicinal properties of mangiferin. Mini Rev Med Chem 12: 412-25.

26. Severi JA, Lima ZP, Kushima H, Monteiro AR, Brito S, et al. (2009) Polyphenols with Antiulcerogenic Action from Aqueous Decoction of Mango Leaves (Mangifera indica L.). Molecules 14: 1098-110.

27. Dar A, Faizi S, Naqvi S, Roome T, Zikr-ur-Rehman S, et al. (2005) Analgesic and antioxidant activity of mangiferin and its derivatives: the structure activity relationship. Biol Pharm Bull 28: 596-600.

28. Pardo-Andreu GL, Delgado R, Nunez-Selles AJ, Vercesi AE (2006) Dual mechanism of mangiferin protection against iron-induced damage to 2-deoxyribose and ascorbate oxidation. Pharmacol Res 53: 253-60.

29. Pardo-Andreu GL, Sánchez-Baldoquín C, Avila-González R, Yamamoto ET, Revilla A, et al. (2006) Interaction of Vimang (Mangifera indica L. extract) with Fe(III) improves its antioxidant and cytoprotecting activity. Pharmacol Res 54: 389-95.

30. Pardo-Andreu GL, Sánchez-Baldoquín C, Avila-González R, Delgado R, Naal, Z et al. (2006) Fe(III) improves antioxidant and cytoprotecting activities of mangiferin. Eur J Pharmacol 547: 31-6.

31. Pardo-Andreu GL, Delgado R, Núñez-Sellés AJ, Vercesi AE (2006) Mangifera indica L. extract (Vimang) inhibits 2-deoxyribose damage induced by Fe (III) plus ascorbate. Phytother Res 20: 120-4.

32. Karuppanan M, Krishnan M, Padarthi P, Namasivayam E (2014) Hepatoprotective and Antioxidant Effect of Mangifera Indica Leaf Extracts against Mercuric Chloride-induced Liver Toxicity in Mice. Euroasian J Hepato-Gastroenterol 4: 18-24.

33. Jain PK, Kharya M, Gajbhjye A (2013) Drug Dev Ind Pharm 39: 1840-50.

34. Campos-Esparza MR, Sanchez-Gomez MV, Malute C (2009) Cell Calcium 45: 358-68.

35. Xia G, Li X, Zhu X, Yin X, Ding H, Qiao Y (2017) Mangiferin protects osteoblast against oxidative damage by modulation of ERK5/Nrf2 signaling. Biochem Biophys Res Commun 491: 807-13.

36. Pan CW, Pan ZZ, Hu JJ, Chen WL, Zhou GY, et al. (2016) Mangiferin alleviates lipopolysaccharide and D-galactosamine-induced acute liver injury by activating the Nrf2 pathway and inhibiting NLRP3 inflammasome activation. Eur J Pharmacol 770: 85-91.

37. Saha S, Rashid K, Sadhukhan P, Agarwal N, Sil PC (2016) Attenuative role of mangiferin in oxidative stress-mediated liver dysfunction in arsenic-intoxicated murines. Biofactors 42: 515-32.

38. Agustini FD, Arozal W, Louisa M, Siswanto S, Soetikno V, Nafrialdi N, Suvatna F (2016) Pharm Bull 54: $1289-97$.

39. Wang B, Wan J, Gong X, Kuang G, Cheng X, et al. (2015) Mangiferin attenuates renal ischemia-reperfusion injury by inhibiting inflammation and inducing adenosine production. Int Immunopharmacol 25: 148-54.

40. Gong X, Zhang L, Jiang R, Ye M, Yin X, Wan J (2013) Anti-inflammatory effects of mangiferin on sepsis-induced lung injury in mice via up-regulation of heme oxygenase-1. J Nutr Biochem 24: 1173-81.

41. Szandruk M, Merwid-Lad A, Szelag A (2018) The impact of mangiferin from Belamcanda chinensis on experimental colitis in rats. Inflammopharmacology 26: $571-81$.

42. Huang H, Tan Z, Deng J, Liang Q, Nong Y, Song N (2011) Zhongguo Zhong Yao Za Shi 36: 443-5.

43. Bock C, Ternes W (2010) The phenolic acids from bacterial degradation of the mangiferin aglycone are quantified in the feces of pigs after oral ingestion of an extract of Cyclopia genistoides (honeybush tea). Nutr Res 30: 348-57.

44. Sanugul K, Akao T, Li Y, Kakiuchi N, Nakamura N, Hattori M (2005) Isolation of a human intestinal bacterium that transforms mangiferin to norathyriol and inducibility of the enzyme that cleaves a C-glucosyl bond. Biol Pharm Bull 28: 1672-8.

45. Zhang QJ, Yue L (2016) Inhibitory Activity of Mangiferin on Helicobacter Pylori-Induced Inflammation in Human Gastric Carcinoma Ags Cells. Afr J Tradit Compliment Altern Med 14: 263-71.

46. Castillo-Juarez I, Gonzalez V, Jaime-Aguilar H, Martinez G, Linares E, Bye R, Romero I (2009) Anti-Helicobacter pylori activity of plants used in Mexican traditional medicine for gastrointestinal disorders. J Ethnopharmacol 122: 402-5.

47. Morais TC, Lopes SC, Carvalho KMMB, Arruda BR, de Souza FTC, et al. Mangiferin, a natural xanthone, accelerates gastrointestinal transit in mice involving cholinergic mechanism. (2012) World J Gastroenterol 18: 3207-14.

48. Carvalho AC, Guedes MM, de Souza AL, Trevisan MT, Lima AF, et al. (2007) Gastroprotective effect of mangiferin, a xanthonoid from Mangifera indica, against gastric injury induced by ethanol and indomethacin in rodents. Planta Med 73: 1372-6.

49. Kakino M, Izuta H, Ito T, Tsuruma K, Araki Y, et al. (2010) Agarwood induced laxative effects via acetylcholine receptors on loperamide-induced constipation in mice. Biosci Biotechnol Biochem 74: 1550-5.

50. Wang X, Gu Y, Ren T, Tian B, Zhang Y, et al. (2013) Increased absorption of mangiferin in the gastrointestinal tract and its mechanism of action by absorption enhancers in rats. Drug Dev Ind Pharm 39: 1408-13.

51. Mahmoud-Awny M, Attia AS, Abd-Ellah MF, El-Abhar HS (2015) Mangiferin Mitigates Gastric Ulcer in Ischemia/ Reperfused Rats: Involvement of PPAR- $\gamma$, NF- $\kappa \mathrm{B}$ and Nrf2/HO-1 Signaling Pathways. PLoS One 10: e0132497.

52. Severi JA, Lima ZP, Kushima H, Brito AR, Santos LC, et al. (2009) Polyphenols with antiulcerogenic action from aqueous decoction of mango leaves (Mangifera indica L). Molecules 14: 1098-110.

53. Somani S, Zambad S, Modi K (2016) Mangiferin attenuates DSS colitis in mice: Molecular docking and in vivo approach. Chem Biol Interact 253: 18-26.

54. Morais TC, Arruda BR, de Sousa Mogalhaes H, Trevisan MT, de Araujo VD, Rao VS, Santos FA (2014) Naunyn Schmiedebergs Arch Pharmacol 388: 531-8.

55. Dou W, Zhang J, Ren G, Ding L, Sun A, et al. (2014) Mangiferin attenuates the symptoms of dextran sulfate sodium-induced colitis in mice via NF- $\kappa B$ and MAPK signaling inactivation. Int Immunopharmacol 23: 170-8.

56. Herrera-Cazares LA, Hernández-Navarro F, Ramírez-Jiménez AK, Campos-Vega R, Reyes-Vega ML, et al. (2017) Mango-bagasse functional-confectionery: vehicle for enhancing bioaccessibility and permeability of phenolic compounds. Food Funct 8: 3906-16. 
57. Jyotshna KP, Shanker K (2016) Mangiferin: A review of sources and interventions for biological activities. Biofactors 42: 504-14.

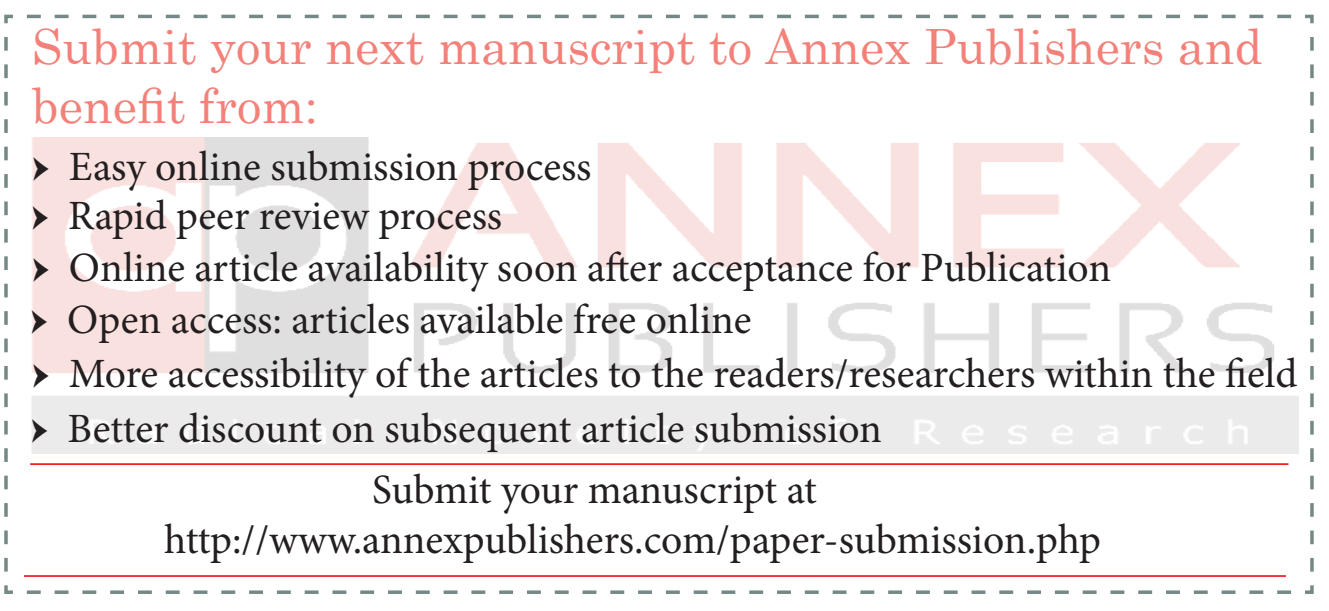

